\title{
An Efficient Algorithm for Workflow Scheduling in the Clouds Based on Differential Evolution Method
}

\author{
Toan Phan Thanh ${ }^{1}$, Loc Nguyen The ${ }^{2}$, Said Elnaffar ${ }^{3}$ \\ ${ }^{1}$ Faculty of Technology Education, Hanoi National University of Education, Ha Noi, Viet Nam \\ ${ }^{2}$ Faculty of Information Technology, Hanoi National University of Education, Ha Noi, Viet Nam \\ ${ }^{3}$ School of Engineering, Computer Science Department, American University of RAK, Ras al Khaimah, UAE \\ Email address: \\ pttoan@hnue.edu.vn (T. P. Thanh), locnt@hnue.edu.vn (L. N. The), said.elnaffar@gmail.com (S. Elnaffar)
}

\section{To cite this article:}

Toan Phan Thanh, Loc Nguyen The, Said Elnaffar. An Efficient Algorithm for Workflow Scheduling in the Clouds Based on Differential Evolution Method. American Journal of Computer Science and Technology. Vol. 1, No. 1, 2018, pp. 24-30.

doi: $10.11648 /$ j.ajcst.20180101.14

Received: October 27, 2017; Accepted: December 4, 2017; Published: January 2, 2018

\begin{abstract}
The Cloud is a computing platform that provides on-demand access to a shared pool of configurable resources such as networks, servers, storage that can be rapidly provisioned and released with minimal management effort from clients. At its core, Cloud computing focuses on manimizing the effectiveness of the shared resources. Therefore, workflow scheduling is one of the challenges that the Cloud must tackle especially if a large number of tasks are executed on geographically distributed servers. The Cloud is comprised of computational and storage servers that aim to provision efficient access to remote and geographically distributed resources. To that end, many challenges, specifically workflow scheduling, are yet to be solved such. Despite it has been the focus of many researchers, a handful efficient solutions have been proposed for Cloud computing. In this work, we propose a novel algorithm for workflow scheduling that is derived from the Opposition-based Differential Evolution method, MODE. This algorithm not only ensures fast convergence but also averts getting trapped in local extrema. Our simulation experiments Cloud Sim show that MODE is superior to its predecessors. Moreover, the deviation of its solution from the optimal one is negligible.
\end{abstract}

Keywords: Workflow Scheduling, Opposition-Based Differential Evolution, Cloud Computing, Differential Evolution

\section{Introduction}

The Cloud is a computing platform that provides convenient, on-demand access to a shared pool of configurable computing resources such as networks, servers, storage that can be rapidly provisioned and released with minimal management effort on clients. At its core, Cloud computing focuses on maximizing the effectiveness of the shared resources. Therefore, workflow scheduling is one of the challenges that the Cloud must tackle especially if a large number of tasks are executed on geographically distributed servers. This requires a reasonable scheduling algorithm in order to minimize a task completion time (makespan).

Our work described in this paper is a new attempt to introduce a more efficient scheduling algorithm. Specifically, we introduce a Modified version of the Opposition-based Differential Evolution (ODE) method [19], which we call MODE. Via experiments, we demonstrate its effectiveness and performance in comparison with other algorithms.

The rest of the paper is organized as follows. Section II reviews some of the related works germane to workflow scheduling algorithms. Section III describes the Oppositionbased Differential Evolution (ODE) method. Section IV describes the computation and communication model on which Cloud tasks operate. Based on this model, Section V presents our proposed scheduling algorithm MODE (Modified ODE). Section VI describes the experiments we conducted using the simulation tool CloudSim [1] in order to evaluate the proposed algorithm. Section VII concludes our paper and sketches future work.

\section{Related Work}

A workflow is a sequence of connected tasks. Workflow 
scheduling in Clouds is a challenge because each task needs to be mapped to an appropriate server while allowing that task to satisfy some performance constraints. In general, the scheduling problem, i.e. the mapping of tasks to the computation resources such as servers, is an NP-complete problem [4]. Hence, past works mainly banked on heuristic-based or metaheuristic-based solutions for scheduling workflows.

Some researchers [13] proposed an algorithm for task scheduling based on two-level load balancing in the cloud environment. This algorithm not only meets user's requirements but also provides high resource utilization. The authors also introduced the implementation of an efficient Quality of Service (QoS) based Meta-heuristic method.

Others [15] presented an optimized algorithm for task scheduling based on Hybrid Genetic Algorithms. They considered the QoS requirements like completion time, bandwidth, cost, distance, reliability of different type tasks. They used annealing in their implementation after the selection, crossover and mutation, to improve local search ability of genetic algorithm.

In [16], the authors presented a model for task scheduling in cloud computing to minimize the overall time of execution and transmission. They proposed a particle swarm optimization algorithm to solve task scheduling that is based on small position value rule (SPVPSO). The researchers compared the SPVPSO algorithm with the CR-PSO and L-PSO and found that the SPVPSO algorithm had attained the optimal solution and converged faster in large tasks than the other two algorithms.

Another work [17] proposed a hierarchical scheduling algorithm which satisfied the Service Level Agreement with quick response from the service provider. The authors used the response time as a Quality of Service (QoS) metric to prioritize the execution of jobs by estimating their completion times.

Another paper [18] presented an optimized algorithm for task scheduling in cloud computing based on Activity-Based Costing (ABC). This algorithm assigns a priority level for each task and uses cost drivers. The priority is estimated based on four major factors: time, space, resources and profit.

Pandey [9] presented a scheduling algorithm (PSO_H) to minimize the cost of the execution. However, instead of finding the schedule which has a minimum cost, $\mathrm{PSO}_{-} \mathrm{H}$ looked for the schedule that minimizes the execution cost at the nearest server. The author compared the PSO_H algorithm with the Random and Round Robin algorithm and showed that the optimal solution of PSO_H algorithm is better than two matching algorithms.

\section{Opposition-Based Differential Evolution (ODE)}

Opposition-based Differential Evolution (ODE) [19] is an evolutionary optimization technique that consists of two main steps: population initialization and producing new generations by genetic operations such as selection, crossover and mutation. ODE enhances these two steps by considering the opposite point of individuals in the population.

\subsection{Opposition-Based Learning}

The concept of Opposition-Based Learning (OBL) was original introduced by Tizhoosh [20]. The main idea behind OBL is the simultaneous consideration of an estimate and its corresponding opposite estimate in order to achieve a better approximation for the current candidate solution.

Definition 1 (Opposite Number): Let $\mathrm{x} \in[\mathrm{a}, \mathrm{b}]$ be a real number, then the opposite number $\bar{x}$ is defined as

$$
\bar{x}=a+b-x
$$

Definition 2 (Opposite Point in n-Dimensional Space): Similarly, the above definition can be extended for higher dimensions as follows:

Let $\mathrm{P}\left(\mathrm{x}_{1}, \mathrm{x}_{2}, \ldots, \mathrm{x}_{\mathrm{D}}\right)$ be an D-dimemsional vector, where $\mathrm{x}_{\mathrm{i}}$ $\in\left[\mathrm{a}_{\mathrm{i}}, \mathrm{b}_{\mathrm{i}}\right] ; \mathrm{i}=1,2, \ldots, \mathrm{D}$. The opposite of $\mathrm{P}$ is defined by: $\bar{P}=\left(\bar{x}_{1}, \bar{x}_{2}, \ldots, \bar{x}_{D}\right)$ where

$$
\overline{x_{l}}=a_{i}+b_{i}-x_{i}
$$

The opposition-based optimization can be defined as follows:

Let $\mathrm{P}=\left(\mathrm{x}_{1}, \mathrm{x}_{2}, . ., \mathrm{x}_{\mathrm{D}}\right)$ be a point in D-dimensional space, and $\mathrm{f}($.$) is the fitness function which is used to measure the$ candidate's fitness. According to the definition of opposite point, $\bar{P}=\left(\bar{x}_{1}, \bar{x}_{2}, \ldots, \bar{x}_{D}\right)$ is the opposite of $\mathrm{P}=\left(\mathrm{x}_{1}, \mathrm{x}_{2}, . ., \mathrm{x}_{\mathrm{D}}\right)$. Now if $f(\bar{P}) \leq f(P)$, then point $\mathrm{P}$ can be replaced by $\bar{P}$, otherwise we continue with $\mathrm{P}$.

\subsection{Opposition-Based Population Initialization}

Using the definition of opposite points, the oppositionbased initialization for the ODE can be described as follows:

Procedure: Opposition-Based Population Initialization

1. Random initialization of population $\mathrm{P}\left(\mathrm{x}_{1}, \mathrm{x}_{2}, \ldots, \mathrm{x}_{\text {PopSize }}\right)$

2. Calculate opposite population by equal $(2)$

3. $\mathrm{OP} \leftarrow$ Opposite $(\mathrm{P})$

4. Selecting the PopSize fittest individuals from $\{P \cup O P\}$ as initial population.

\subsection{Opposition-Based Differential Evolution Algorithm}

By combining the Opposition-Based Learning method and the Differential Evolution algorithm, the ODE algorithm can be described as follows:

Algorithm:Opposition-Based Differential Evolution

1. Call Procedure: Opposition-Based Population Initialization

2. for $\mathrm{i}=1$ to PopSize do

3. $\mathrm{f}_{\mathrm{i}} \leftarrow$ fitness $\left(\mathrm{x}_{\mathrm{i}}\right)$

4. while (criteria is not satisfied) do

5. for $\mathrm{i}=1$ to PopSize do

6. $\mathrm{r} 1, \mathrm{r} 2 \leftarrow \operatorname{Random}(1$, PopSize $)$

7. $\mathrm{F} \leftarrow \operatorname{Random}(0,1)$

8. $\mathrm{v}_{\mathrm{i}}(\mathrm{t}) \leftarrow$ pbest $+\mathrm{F} \times\left(\mathrm{x}_{\mathrm{r} 1}-\mathrm{x}_{\mathrm{r} 2}\right)$

9. for $\mathrm{j}=0$ to $\mathrm{D}$ do 
10. $u_{i, j}=\left\{\begin{array}{l}v_{i, j} \text { nếu } \operatorname{rand}_{i, j} \leq C R \text { hoặc } i=I_{\text {rand }} \\ p_{i, j} \text { nếu } \operatorname{rand}_{i, j} \geq C R \text { hoặc } i \neq I_{\text {rand }}\end{array}\right.$

11. end for

12. if $\left(\mathrm{f}\left(\mathrm{u}_{\mathrm{i}}(\mathrm{t})\right) \leq \mathrm{f}\left(\mathrm{x}_{\mathrm{i}}(\mathrm{t})\right)\right)$

13. $\mathrm{x}_{\mathrm{i}}(\mathrm{t}+1)=\mathrm{u}_{\mathrm{i}}(\mathrm{t})$

14. end if

15. end for

16. rand $\leftarrow \operatorname{Random}(0,1)$

17. if $\left(\right.$ rand $<\mathrm{J}_{\mathrm{r}}$ )

18. Calculating Opposite Population OP(PopSize)

19. Fitness Evaluation

20. Selecting PopSize Fittest Individuals from \{Current Population, OP

21.end if

22. end while

\section{Problem Formulation}

We denote the workflow as a Directed Acyclic Graph (DAG) represented by $\mathrm{G}=(\mathrm{V}, \mathrm{E})$, where:

1. $\mathrm{V}$ is set of vertex, each vertex represents a task

2. $\mathrm{T}=\left\{\mathrm{T}_{1}, \mathrm{~T}_{2}, \ldots, \mathrm{T}_{\mathrm{M}}\right\}$ is the set of tasks, $\mathrm{M}$ is the number of tasks

3. E represents the data dependencies between these tasks. The edge $\left(T_{i}, T_{j}\right) \in E$ means the task $T_{i}$ is the father of the task $T_{j}$, the data produced by $T_{i}$ will be consumed by the task $T_{j}$.

4. The Cloud's computation resources are a set of servers $\mathrm{S}=\left\{\mathrm{S}_{1}, \mathrm{~S}_{2}, \ldots, \mathrm{S}_{\mathrm{N}}\right\} . \mathrm{N}$ is the number of servers.

5. Each task $T_{i}$ can be executed by any server $S_{j} \in S$, and $\mathrm{S}_{\mathrm{i}}$ has to handle the whole the workload of $\mathrm{T}_{\mathrm{i}}$

6. The computation of task $\mathrm{T}_{\mathrm{i}}$ denoted by $\mathrm{W}_{\mathrm{i}}$ (flopfloating point operations)

7. $\mathrm{P}\left(\mathrm{S}_{\mathrm{i}}\right)$ : the computation power of the server $\mathrm{S}_{\mathrm{i}}(\mathrm{MI} / \mathrm{s}$ : million instructions/second)

8. The bandwidth $B\left(S_{i}, S_{j}\right)$ between server $S_{i}$ and server $S_{j}$ is represented by the function $\mathrm{B}(): \mathrm{S} \times \mathrm{S} \rightarrow \mathrm{R}^{+}$. We assume that $B\left(S_{i}, S_{i}\right)=\infty$ and $B\left(S_{i}, S_{j}\right)=B\left(S_{j}, S_{i}\right)$

9. $D_{i j}$ : data produced by task $T_{i}$ and consumed by task $T_{j}$.

Each scheduling plan can be represented by the function $f(): \mathrm{T} \rightarrow \mathrm{S}$ where $f\left(T_{i}\right)$ is the server that handles the task $T_{i}$

Based on the above assumptions we have:

10. The execution time of the task $T_{i}$ is

$$
\frac{W_{i}}{P\left(f\left(T_{i}\right)\right)}
$$

11. The communication time between the task $T_{i}$ and $T_{j}$ is

$$
\frac{D_{i j}}{B\left(f\left(T_{i}\right), f\left(T_{j}\right)\right)}
$$

Formally, we need to minimize the execution time, called makespan, of the workflow:

makespan $\rightarrow$ min

where makespan is the time difference between the start and finish of a sequence of workflow's tasks.

\section{Proposed Algorithm}

\subsection{Particle Representation}

In the proposed scheduling algorithm, the solution is represented as a vector of length equal to the number of tasks. The value corresponding to each position $i$ in the vector represents the server to which task $i$ was executed.

Example 1

Consider a workflow with a set of tasks $\mathrm{T}=\left\{T_{1}, T_{2}, T_{3}, T_{4}\right.$, $\left.T_{5}\right\}$, a set of servers $\mathrm{S}=\left\{S_{1}, S_{2}, S_{3}\right\}$. So the particle $x_{i}^{k}=[1$; $2 ; 1 ; 3 ; 2]$ gives us the following scheduling plan:

$\begin{array}{lllll}\mathrm{T}_{1} & \mathrm{~T}_{2} & \mathrm{~T}_{3} & \mathrm{~T}_{4} & \mathrm{~T}_{5} \\ \mathrm{~S}_{1} & \mathrm{~S}_{2} & \mathrm{~S}_{1} & \mathrm{~S}_{3} & \mathrm{~S}_{2}\end{array}$

In that scheduling plan, tasks T1 and T3 will be executed by the server $\mathrm{S} 1$, tasks $\mathrm{T} 2$ and $\mathrm{T} 5$ are assigned to the server $\mathrm{S} 2$ and task T4 is handled by server S3.

\subsection{Method to Calculate the Opposite of Individuals}

The design of ODE entails calculating the opposite of individuals in the population, which can be carried out as follows:

Let $\mathrm{a}=\operatorname{Max}\left\{\mathrm{P}\left(\mathrm{S}_{\mathrm{i}}\right)\right\} ; \forall \mathrm{i}=1,2, . ., \mathrm{N}$
$\mathrm{b}=\operatorname{Min}\left\{\mathrm{P}\left(\mathrm{S}_{\mathrm{i}}\right)\right\} ; \forall \mathrm{i}=1,2, . ., \mathrm{N}$

Assuming that the particle $\mathrm{x}_{\mathrm{i}}=\left(\mathrm{S}_{\mathrm{i} \pi(1)}, \mathrm{S}_{\mathrm{i} \pi(2)}, \ldots, \mathrm{S}_{\mathrm{i} \pi(\mathrm{M})}\right) ; \mathrm{S}_{\mathrm{i} \pi(\mathrm{j})}$ $\in \mathrm{S}, \forall \mathrm{j}=1,2, . ., \mathrm{M}$; the opposite of $\mathrm{x}_{\mathrm{i}}$, denoted by $\bar{x}_{l}$, will be calculated as follows:

$$
\bar{x}_{l}=\left(\bar{S}_{i \pi(1)}, \bar{S}_{i \pi(2)}, \ldots, \bar{S}_{i \pi(M)}\right)
$$

where:

$$
\bar{S}_{i \pi(j)}=a+b-S_{i \pi(j)} ; \forall j=1,2, \ldots, M
$$

We we subsequently assign the value corresponding to each position $j$ of vector $\bar{x}_{l}$ by identifying the server which has a computation power closer to $\bar{S}_{i \pi(j)}$ than any other server

$$
\overline{x_{l \jmath}} \leftarrow k \text { nếu }\left|P\left(S_{k}\right)-\bar{S}_{i \pi(j)}\right| \leq\left|P\left(S_{r}\right)-S_{i \pi(j)}\right| \forall S_{r}
$$

Algorithm: OP_Algorithm

Input: population $\mathrm{p}=\left(\mathrm{x}_{1}, \mathrm{x}_{2}, \ldots, \mathrm{x}_{\mathrm{PopSize}}\right)$

Output: opposite of population OP

1. $\mathrm{a} \leftarrow \operatorname{Max}\left\{\mathrm{P}\left(\mathrm{S}_{\mathrm{i}}\right)\right\} ; \forall \mathrm{i}=1,2, \ldots, \mathrm{N}$

2. $\mathrm{b} \leftarrow \operatorname{Min}\left\{\mathrm{P}\left(\mathrm{S}_{\mathrm{i}}\right)\right\} ; \forall \mathrm{i}=1,2, \ldots, \mathrm{N}$

3. for $\mathrm{i}=1$ to PopSize do

4. $\bar{x}_{\imath} \leftarrow$ opposite $\left(x_{i}\right)$; by equation (5)

5. assign the identity of the server to each position $\mathrm{j}$ of vector $\bar{x}_{l}$ by equation (6)

6. end for return $\mathrm{OP}$

\subsection{Rank-Based Roulette Wheel Selection}

Rank-based roulette wheel selection is the selection strategy where the probability of a particle being selected is based on its fitness rank relative to the entire population. Rank-based selection schemes first sort individuals in the 
population according to their fitness and then computes the selection probabilities according to their ranks rather than the fitness values. Rank-based selection uses a function to map the indices of individuals in the sorted list to their selection probabilities. The rank for an individual may be scaled linearly using the following formula:

$\operatorname{rank}($ pos $)=2-S P+\left(2 \times(S P-1) \times \frac{\text { pos }-1}{\text { PopSize }-1}\right) ;$ where $1.0 \leq$

$$
\mathrm{SP} \leq 2.0
$$

The algorithm to selected individuals from population by Rank-based Roulette Wheel Selection can be described as follows:

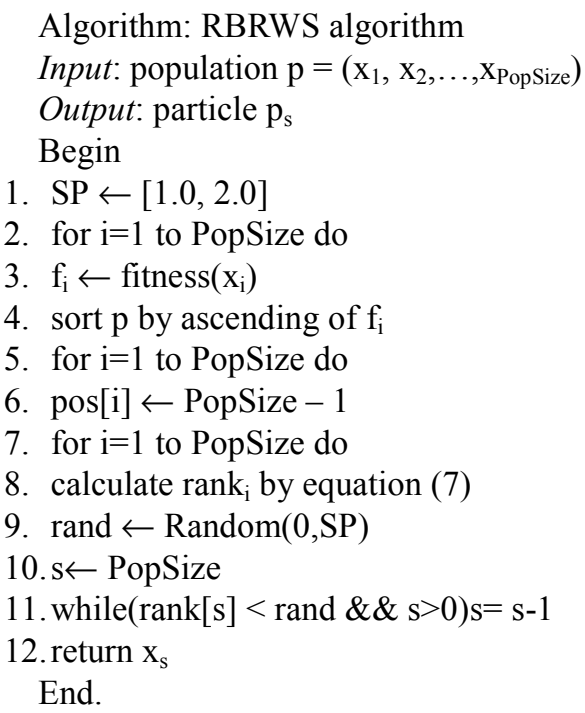

\subsection{The MODE Algorithm}

The MODE algorithm can be described as follows:

\section{Algorithm MODE ( )}

Input: T, S, size of workload $\mathrm{W}[1 \times M], \mathrm{P}[1 \times N], \mathrm{B}[N \times N]$,

$\mathrm{D}[M \times M]$, the number of particle $N o P$

Output: the best position gbest

1. Call procedure: Opposition-Based Population Initialization

2. while(criteria is not satisfied)do

3. for $\mathrm{i}=1$ to PopSize do

4. selecting $\mathrm{p}_{1}$ from population by RBRWS algorithm

5. selecting $\mathrm{p}_{2}$ from population by RBRWS algorithm

6. $\mathrm{F} \leftarrow$ Random $(1,0)$

7. $\mathrm{v}_{\mathrm{i}} \leftarrow$ pbest $+\mathrm{F} \times\left(\mathrm{p}_{1}-\mathrm{p}_{2}\right)$

8. assign the identity of the server to each position $\mathrm{j}$ of vector $\mathrm{v}_{\mathrm{i}}$ by equation (4)

9. $\operatorname{rand}_{\mathrm{i}, \mathrm{j}} \leftarrow \operatorname{Random}(0,1)$

10. $\mathrm{I}_{\text {rand }} \leftarrow \operatorname{random}(1, \mathrm{M})$

11. $u_{i, j}=\left\{\begin{array}{l}v_{i, j} \text { nếu rand } i, j \leq C R \text { hoặc } i=I_{\text {rand }} \\ x_{i, j} \text { nếu rand } i, j \geq C R \text { hoặc } i \neq I_{\text {rand }}\end{array}\right.$

12. if $\left(\operatorname{makespan}\left(\mathrm{u}_{\mathrm{i}}\right)<\operatorname{makespan}\left(\mathrm{x}_{\mathrm{i}}\right)\right)$

13. $\mathrm{x}_{\mathrm{i}} \leftarrow \mathrm{u}_{\mathrm{i}}$

14. if $\left(\right.$ rand $\left.<\mathrm{J}_{\mathrm{r}}\right)$

15. Calculating Opposite Population OP by OP_Algorithm 16. Fitness Evaluation
17. Selecting PopSize Fittest Individuals from \{Current Population, OP

18. End while

Return gbest;

In the Initialization step, we randomly generate a population of PopSize individuals, and calculate the opposite of individuals in the population using equation (5) and (6), then select the Popsize fittest individuals from current population and their counterpart opposites.

In each iteration, MODE selects two individuals from population according to the Rank-based Roulette Wheel Selection method. According to the mutation operator, a mutant vector is generated by adding the weighted difference between two selected vector of the target population individuals to a third one as follows :

$$
\mathrm{v}_{\mathrm{i}} \leftarrow \text { pbest }+\mathrm{F} \times\left(\mathrm{p}_{1}-\mathrm{p}_{2}\right)
$$

where $\mathrm{p} 1, \mathrm{p} 2$ are two individuals selected by rank-based roulette wheel selection method, and pbest is the best individual in the population.

After the mutation phase, the crossover operator is applied to obtain the trail vector $\mathrm{u}_{\mathrm{i}}$ using the following equation :

$$
u_{i, j}=\left\{\begin{array}{l}
v_{i, j} \text { if } \operatorname{rand}_{i, j} \leq C R \text { or } i=I_{\text {rand }} \\
x_{i, j} \text { if } \operatorname{rand}_{i, j} \geq C R \text { or } i \neq I_{\text {rand }}
\end{array}\right.
$$

Where $\operatorname{rand}_{i, j}$ is the $j^{\text {th }}$ independent random number uniformly distributed in the range of $[0,1]$. Also $I_{\text {rand }}$ is a random number in the range of $[1, \mathrm{M}]$. CR is a user defined crossover factor in the range $[0,1]$.

Now, we need to decide whether the trail vector $u_{i}$ should be a member of the population of the next generation, it is compared with the target individual $\mathrm{x}_{\mathrm{i}}$. Finally, the selection is based on the survival of the fitness as follows :

$$
x_{i}=\left\{\begin{array}{c}
u_{i}, \text { if makespan }\left(u_{i}\right)<\text { makespan }\left(x_{i}\right) \\
x_{i}, \text { otherwise }
\end{array}\right\}
$$

After the crossover phase, we calculate the opposite of individuals in the population by equation (5) and (6). The fitness of individuals is evaluated, then the PopSize fittest individuals are selected for the next generation.

\section{Evaluation}

We conducted some experiments in order to compare the performance of the MODE against the PSO_H [9] and Random [12]. Our experimental setup consists of a computer with Intel Core i5 $2.2 \mathrm{GHz}$, RAM 4GB, and Windows 7 Ultimate. The experiments were carried out using the simulation tool CloudSim [14] and the packet library Jswarm [1] and Java.

\subsection{Data}

We use both random and real world instances in our experiments using the following data sets:

The computation power of the servers and the bandwidth of connections between servers are collected from a Cloud 
provider such as Amazon [2] and its Web site (exp. http://aws.amazon.com/ec2/pricing)

The sets of working data are collected from Montage project [3]. The instances are divided into 6 groups based on the number of servers $\mathrm{N}$ and the number of tasks $\mathrm{M}$ :

Group 1: $\mathrm{M}=10, \mathrm{~N}=3$; Group 2: $\mathrm{M}=10, \mathrm{~N}=5$; Group 3: $\mathrm{M}=20, \mathrm{~N}=5$; Group 4: $\mathrm{M}=20, \mathrm{~N}=8$;

Group 5: $\mathrm{M}=25, \mathrm{~N}=8$; Group 6: $\mathrm{M}=50, \mathrm{~N}=8$;

We denote the ratio of the number of edges and the number of vertexes of graph $\mathrm{G}$ by:

$$
\alpha=\frac{|E|}{M \times(M-1) / 2}
$$

\subsection{Configuration Parameters}

The Cloud's configuration parameters are chosen as follows:

1. Server's computation power: from 1 to 250 (million instructions/s)

2. Connection bandwidth B: from 10 to 100 (Mbps)

3. Communication data $\mathrm{D}$ : from 1 to 10000 (MB)

The MODE's configuration parameters are:

1. Number of particles $N o P=100 ; \alpha$ : from 0.2 to 0.7 ;
2. Differential amplification factor $\mathrm{F}=0.5$;

3. Crossover probability constant $\mathrm{CR}=0.9$;

4. Jumping rate constant $\mathrm{J}_{\mathrm{r}}=0.3$.

\subsection{Results}

Each problem instance was executed 30 times continuously. The results summarized in Table 1 show that the mean value (column Mean) and standard deviation value (column STD) of MODE are better than those of PSO_H [9] and Random [12] in most of the cases. When the number of servers $(N)$ and the number of tasks $(M)$ are relatively large (i.e. larger scale cloud), for example $M=20$ and $N=8 ; M=25$, $N=8 ; M=50, N=8$, MODE is better than PSO_H and Random with respect to all metrics: mean, standard deviation and best value (column Best).

Figures 1-6 depict the performance of the three algorithms: proposed algorithm MODE, PSO_H [9], and Random [12] where the vertical axis represents the makespan (seconds) of the schedule. For each instance, we compare the best position vector (column BEST), the mean value (column MEAN) and standard deviation value (column STD). At the first instance, MODE even found the optimal solution.

Table 1. Comparison the makespan of MODE with other algorithms.

\begin{tabular}{|c|c|c|c|c|c|c|c|c|c|c|c|}
\hline \multirow{2}{*}{$\mathbf{M}$} & \multirow{2}{*}{$\mathbf{N}$} & \multirow{2}{*}{$\alpha$} & \multicolumn{3}{|c|}{ MODE } & \multicolumn{3}{|c|}{ PSO_H } & \multicolumn{3}{|c|}{ RANDOM } \\
\hline & & & Best & Mean & STD & Best & Mean & STD & Best & Mean & STD \\
\hline 10 & 3 & 0.26 & 16.9 & 17.3 & 0.4 & 16.4 & 20.4 & 2.4 & 21.4 & 28.6 & 3.2 \\
\hline 10 & 5 & 0.26 & 75.5 & 78.2 & 0.9 & 86.0 & 107.5 & 13.2 & 123.3 & 184.1 & 42.4 \\
\hline 20 & 5 & 0.15 & 27.5 & 29.3 & 1.4 & 29.6 & 41.0 & 5.0 & 45.8 & 59.0 & 6.8 \\
\hline 20 & 3 & 0.31 & 32.5 & 33.9 & 0.8 & 33.2 & 41.9 & 4.6 & 47.4 & 65.6 & 7.8 \\
\hline 20 & 8 & 0.31 & 29.9 & 31.7 & 1.2 & 37.1 & 44.7 & 6.1 & 51.6 & 67.6 & 6.8 \\
\hline 50 & 8 & 0.3 & 9.1 & 9.7 & 0.5 & 12.1 & 14.0 & 0.9 & 13.9 & 87.1 & 25.2 \\
\hline
\end{tabular}

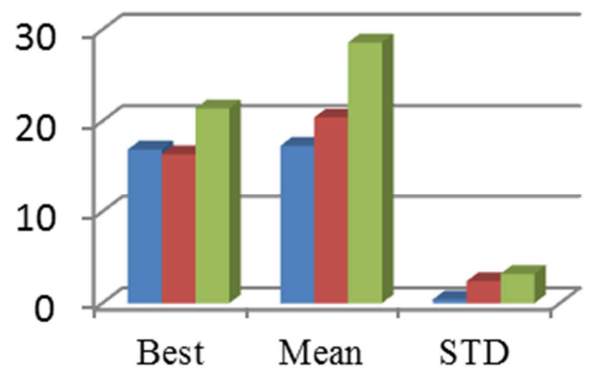

Figure 1. $M=10, N=3$.

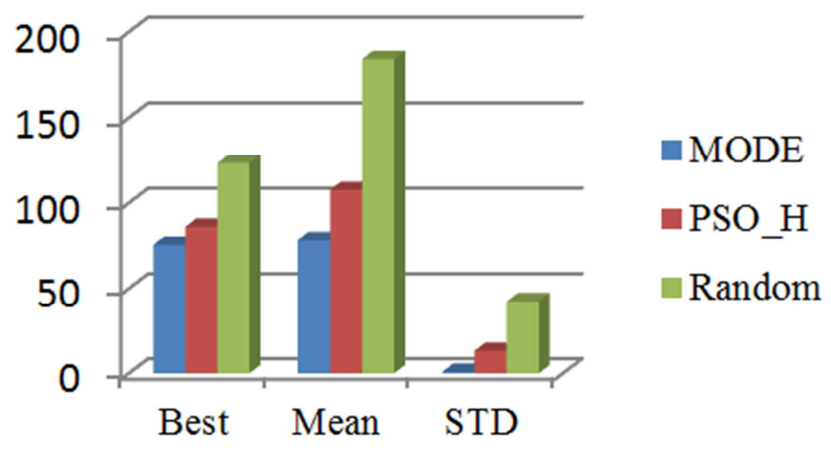

- MODE

-PSO_H

anandom
60

20

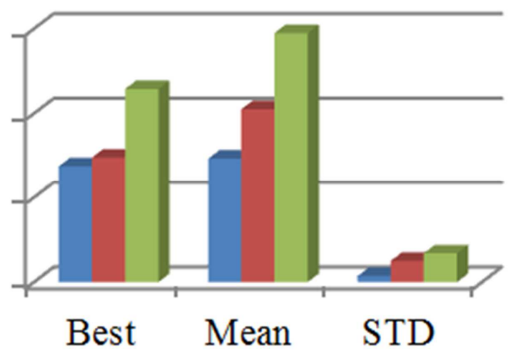

Figure 3. $M=25, N=8$.

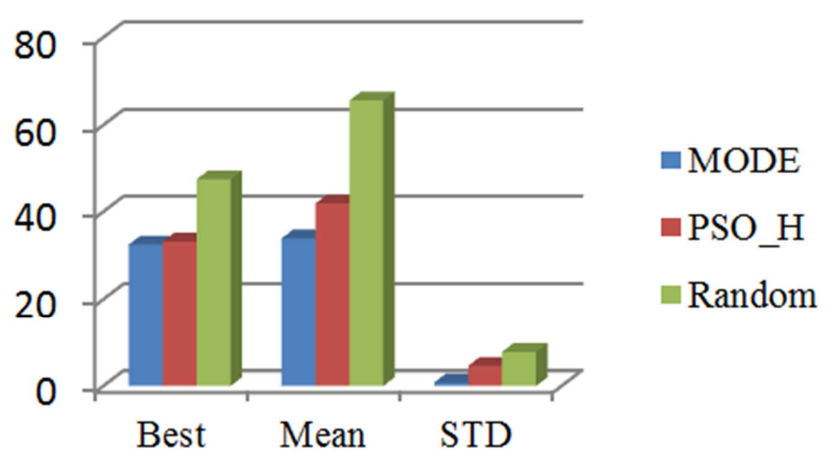

Figure 4. $M=20, N=3$.

- MODE

- $\mathrm{PSO} \_\mathrm{H}$

- Random

Figure 2. $M=10, N=5$. 

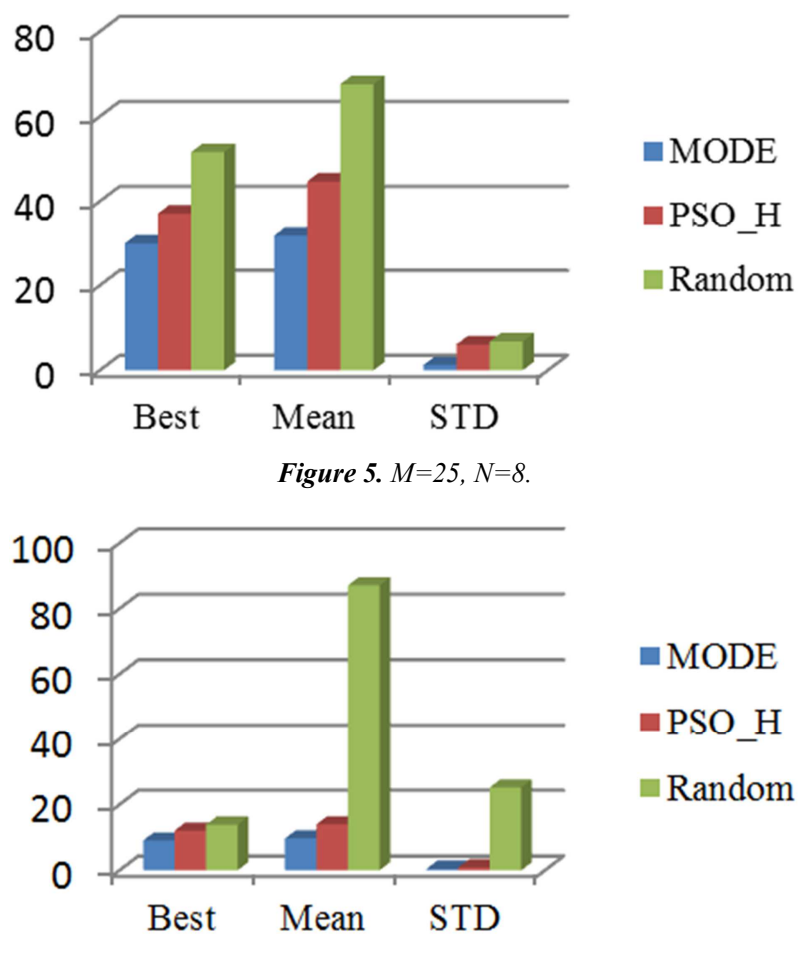

Figure 6. $M=50, N=8$.

\section{Conclusion}

The ultimate goal of any scheduling algorithm is to minimize the execution time. Far from that goal, our proposed algorithm also avoid being trapped on local extrema. The contributions of our paper are:

1. Building a novel approach, represented by the formula to calculate the opposite of individuals in the population.

2. Proposing a new scheduling algorithm named MODE by incorporating the ODE (Opposition-Based Differential Evolution) strategy and Rank-based Roulette Wheel Selection method.

The experimental results show that MODE is superior to its predecessor especially when MODE works in a larger scale Cloud, i.e. the number of servers and tasks are fairly large. In the future, we are planning to improve the MODE algorithm in order to solve bigger instances in a reasonable makespan.

\section{References}

[1] R. N. Calheiros, R. Ranjan, A. Beloglazov, Cesar A. F. De Rose, and R. Buyya, CloudSim: A Toolkit for Modeling and Simulation of Cloud Computing Environments and Evaluation of Resource Provisioning Algorithms, Software: Practice and Experience, volume 41, Number 1, Pages: 23-50, Wiley Press, USA, 2011

[2] J. V. Vliet, F. Paganelli, Programming Amazon EC2, O'Reilly Media, ISBN 1449393683, 2011

[3] http://montage.ipac.caltech.edu
[4] J. D. Ullman, NP-complete scheduling problems, Journal of Computer and System Sciences, pages 384-393, volume 10, issue 3,1975

[5] S. Parsa, R. E. Maleki, RASA: A New Task Scheduling Algorithm in Grid Environment, International Journal of Digital Content Technology and its Applications, volume 3, No. 4, 2009

[6] A. Agarwal, S. Jain, Efficient Optimal Algorithm of Task Scheduling in Cloud Computing Environment, International Journal of Computer Trends and Technology Volume 9, 2014

[7] J. Huang, The Workflow Task Scheduling Algorithm Based on the GA Model in the Cloud Computing Environment, Journal of software, volume 9, 2014

[8] H. Liu, A. Abraham, C. Grosan, A Novel Variable Neighborhood Particle Swarm Optimization for Multiobjective Flexible Job-Shop Scheduling Problems, Proc. of 2nd International Conference on Digital Information Management (ICDIM '07), Volume 1, pages 138-145, 2007.

[9] S. Pandey, L. Wu1, S. M. Guru, R. Buyya1, A Particle Swarm Optimization (PSO)-based Heuristic for Scheduling Workflow Applications in Cloud Computing Environments, Proc. of 24th IEEE International Conference on Advanced Information Networking and Applications (AINA), pages 400-407, 2010

[10] J. Kennedy, R. C. Eberhart, Particle swarm optimization, Proc. of IEEE International Conference on Neural Networks. pages 1942-1948, 1995

[11] A. E. M. Zavala, EVOLVE - A Bridge between Probability, Set Oriented Numerics, and Evolutionary Computation IIA Comparison, A Comparison Study of PSO Neighborhoods, pages 251-295, Springer-Verlag Berlin Heideberg, 2013

[12] M. Mitzenmacher, E. Upfal, Probability and Computing: Randomized Algorithms and Probabilistic Analysis, Cambridge University Press (2005)

[13] Dr. Sudha Sadhasivam, R. Jayarani, Dr. N. Nagaveni, R. Vasanth Ram, Design and Implementation of an efficient Twolevel Scheduler for Cloud Computing Environment, In Proceedings of International Conference on Advances in Recent Technologies in Communication and Computing, 2009

[14] R. Buyya, R. Calheiros, Modeling and Simulation of Scalable Cloud Environment and the Cloud Sim Toolkit: Challenges and Opportunities, IEEE publication 2009, pp1-11

[15] G. Guo-Ning and H. Ting-Lei, Genetic Simulated Annealing Algorithm for Task Scheduling based on Cloud Computing Environment, In Proceedings of International Conference on Intelligent Computing and Integrated Systems, 2010, pp. 60-63

[16] L. Guo, Task Scheduling Optimization in Cloud Computing Based on Heuristic Algorithm, Journal of networks, v.7, No.3, 2012, pp. 547-552

[17] R. Rajkumar, T. Mala, Achieving Service Level Agreement in Cloud Environment using Job Prioritization in Hierarchical Scheduling, Proceeding of International Conference on Information System Design and Intelligent Application, 2012, vol 132, pp 547-554

[18] Q. Cao, W. Gong and Z. Wei, An Optimized Algorithm for Task Scheduling Based On Activity Based Costing in Cloud Computing, In Proceedings of Third International Conference on Bioinformatics and Biomedical Engineering, 2009, pp.1-3 
[19] R. Storn and K. Price, Differential Evolution-A Simple and Efficient Heuristic for Global Optimization over Continuous Spaces, Journal of Global Optimization, 1997, pp. 341-359
[20] H. R. Tizhoosh, Opposition-based learning: A new scheme for machine intelligence, International Conference on Computational Intelligence for Modelling, 2005, pp. 695-701 